\section{THE KINEMATICS OF MACHINERY}

THE study of pure mechanism, a branch of kinematics, in general consists of the solution of the following problems :--Given the mode of connection of two or more points or bodies with each other, required their comparative motion, and conversely given their comparative motion to find their proper connection. Now the comparative motion of two points is determined, as laid down by Willis, when (I) the velocity ratio or the proportion which their velocities bear to each other, and (2) their directional relations, are known; the latter requiring for its complete determination (a) the angle between the directions compared, (b) the angle which the plane containing the two directions makes with a plane fixed in space, and $(c)$ the angle the intersection of the two planes makes with a fixed line on the latter plane. In "Kinematics of Machinery," the English translation by Prof. A. B. W. Kennedy of Prof. Reuleaux's "Theoretische Kinematik," 1 the study is confined within narrower limits, causing the translator not a little difficulty, as he expresses in his preface, to translate the word kinematic, carrying as it does a more extended signification here than on the Continent. Starting with the condition that the change of position is definite at each instant, and determined by the form and connection of the fixed and moving parts, Prof. Reuleaux proceeds to investigate the directional relations of the motion and the arrangements of the parts by which the motion is best brought about without any reference to the idea of velocity.

On turning to the Appendix, pp. $585-589$, we find a most interesting historical collection of the definitions of

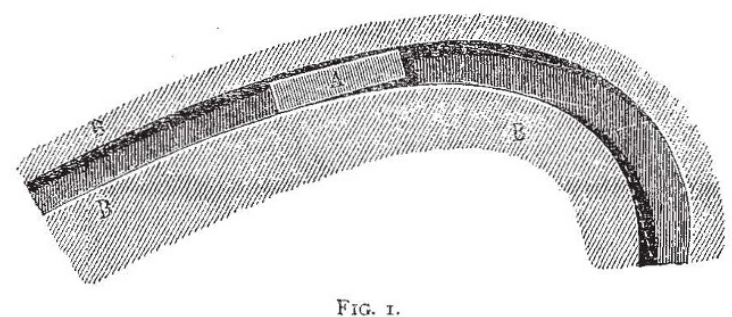

a machine, one of which definitions we remark includes equally an adhesive fly-paper and the red-hot poker of the clown; that given by Reuleaux, p. 35 , is more concise and certainly nearer the point than most of these. "A machine is a combination of resistant bodies so arranged that by their means the mechanical forces of nature can be compelled to do work accompanied by certain determinate motions."

Thus the prevention, by the resistance of the different parts, of all motion other than that desired, as well as the conversion into useful work of as much of the energy expended as its efficiency permits, is the function of the machine. "Those parts of a machine transmitting the forces by which the moving points are caused to limit their motions in the definite and required manner, must be bodies of suitable resistant capacity; the moving parts themselves must belong also to similar bodies." But the determination of the suitable form and sectional area of the resistant parts, though indispensable in the construction of the machine, belongs to another part of the study of machine design, and cannot be included in the kinematic discussion.

We now come to the conception of a pair of elements.

In order that a body B (Fig. I) may prevent all other motion in the body A than that desired, A being

x "The Kinematics of Machinery: Outlines of a Theory of Machines." By F. Reuleaux, Director of and Professor in the Königlichen GewerbeAkademie in Berlin, Member of the Königl. technischen Deputation für Gewerbe. Translated and edited by Alex. B. W. Kennedy, C. E., Professor of Civil and Mechanical Engineering in University College, London. (London: Macmillan and Co., 1876.) assumed to move in a plane, B must be given a form such that it always remains in contact with $\mathrm{A}$ in all its required positions ; when that has been done no other motion can be given to $A$ with respect to $B$. This geometrical form of $\mathrm{B}$ is called the envelope of $\mathrm{A}$, and it is plain that the motion of $B$ with respect to $A$, considered fixed, is the same as that of $A$ with respect to $B$, and that no other motion of $B$ with respect to $A$ is possible; that is, $A$ is also the envelope of $B$. The relation is thus seen to be reciprocal. A combination of this sort is called a pair of elements, and a machine consists solely of such elements, corresponding reciprocally in pairs. "The shaft and the bearing, the screw and the nut are examples of such pairs of elements. We see here that the kinematic elements of a machine are not employed singly, but always in pairs, or, in other words, that the machine cannot so well be said to consist of elements as of pairs of elements. If a kinematic pair of elements be given, a definite motion can be obtained by means of them if one be held fast or fixed in position. The other element is free to be moved, but only in the one particular way allowed by the constitution of the pair."

In order to combine two pairs of elements $a b$ and $c d$, we must unite each element of one pair with one of the elements of the other pair. If this is effected as in Fig. 2, no new motion is obtained, as also when they are in the same straight line; but if $a$ and $d$ are urited in such a manner as not to be parallel or in one straight line, the motion is entirely altered, and any points betwe $b$ and $c$ will describe a curve. In either of these two cases having united $a$ to $d$ and $b$ to $c$, we have only two resistant bodies each limiting and determining the relative motion

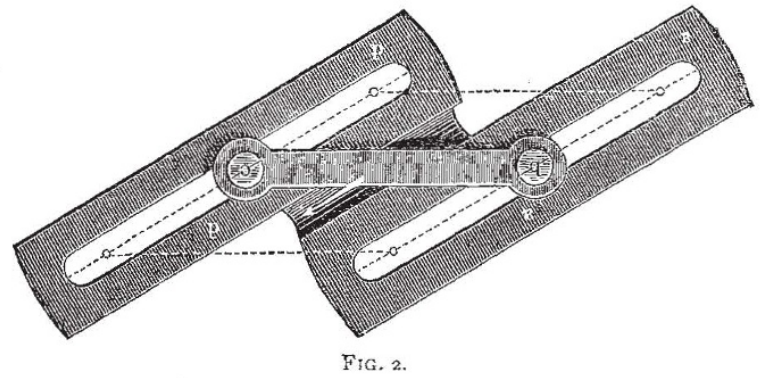

of the other; thus the two pairs of elements are reduced to one pair, in one case with the same, in the other with a different motion.

"Accordingly, the reciprocal combination of the elements of two pairs gives us again a pair of elements which may differ from either of the single pairs of which it is composed." Again, a combination of three, four, or more pairs of elements may be made, each element of each pair being combined with one element of another pair, thus forming a linkage returning upon itself, or a socalled closed kinematic chain. Fig. 3 shows this combination. As a good example of this, the beam, connecting rod and crank of a beam-engine may be taken ; $a$ and $b$ are the Plummer blocks of the crank shaft and main centre rigidly connected together by the bar $a h$, which represents in the engine the rigid connection of these two by the frame, supports, and bed; $b$ the crank-shaft rigidly connected by the crank with the crank-pin $c$; $d e$ the connecting rod rigidly connecting the crank pin $c$ with the gudgeon $f$, and lastly, the beam $e h$ rigidly connecting the gudgeon $f$ with the main centre $g$. In this closed chain of four pairs of elements the only motions of each part with respect to $a h$ regarded as fixed are readily seen. "Thus we are led on to the result that "the mechanism is a closed kinematic chain ; the kinematic chain is compound or simple, and consists of kinematic pairs of elements these carry the envelopes required for the motion which the bodies in contact must have, and by these all motions 
other than those desired in the mechanism are prevented." When one of the mechanical forces of nature, such as that of falling water, moving air, or expanding steam, is applied to one of the movable links in such a manner as to cause it to change its position, mechanical work is performed accompanied by certain determinate motions, and the whole is called a machine. The relative motion of two bodies in a plane is next considered, and the conceptions of the instantaneous centre and of centroids

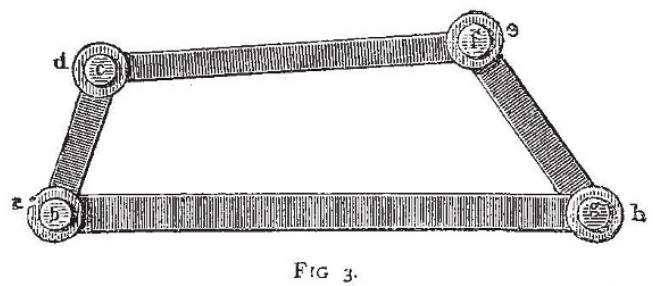

introduced. At each instant of the motion in a plane of one body with respect to another considered as fixed, the motion can be accurately represented by a rotation in the plane about a fixed point, which, however, in each succeeding instant may occupy a different position; this point is called the instantaneous centre, and the positions it occupies in successive instants trace the centroid. Space will not permit us to show the formation of the reciprocal centroid, or how the motion of the moving body can be represented at each instant by the rolling on one another of the centroids, and the motion of any points connected rigidly with the moving body determined when the centroids are known, but the example given as illustrating the determination of relative motion from the known centroids will speak for itself. The centroids given are a circle and straight line which roll on each other. All points rigidly connected with the circle

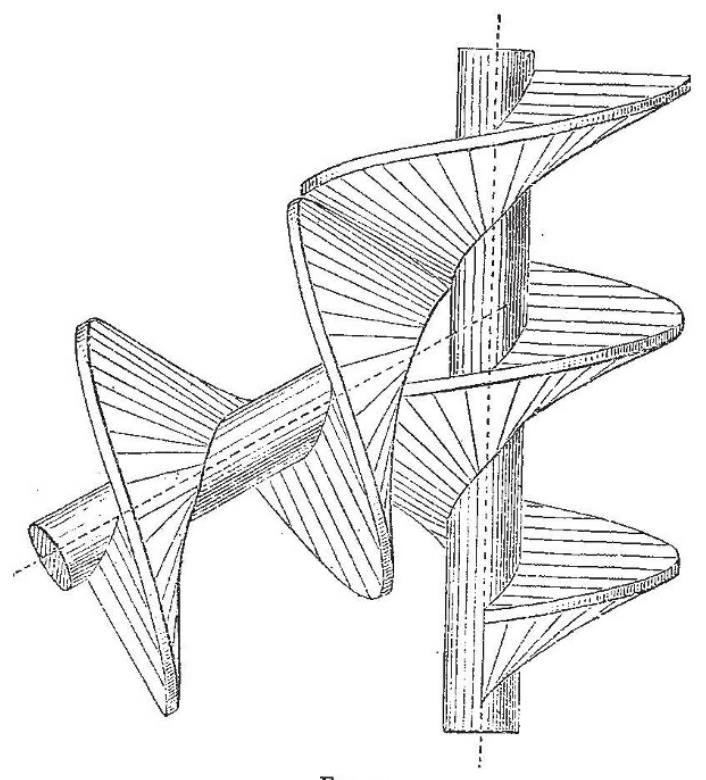

FIG. 4.

describe trochoids, the line being regarded as fixed; all points rigidly connected with the straight line describe involutes, the circle being considered fixed, and all these paths are determinate, and can be constructed if the position of the moving point with respect to the moving element, circle or line, and the centroids, the line and circle are given.

From motion in a plane and the determination of centroids. we pass to motion in space. If the position of three points in a rigid body not in the same straight line are known, the position of any other point in it may be determined from them, and if the three points are fixed in space the body is also fixed. Thus, to determine the relative motion of two rigid bodies in space, we have only to consider the motion of two triangles fixed one in each of them ; or the motion of one triangle fixed in the moving body with respect to the other reduced to rest. The change of position of the moving triangle may take place in many ways, but it may in every case be effected by its translation parallel to itself in a line joining the old and new positions of one of its angular points, and then by a rotation about an axis through the new position of that angular point. Thus any change of position of a rigid body may be effected by a simple translation and a simple translation about an axis. The simplest case is when the transiation takes place along a line parallel to the axis of rotation, when, if the change of position of the moving body be taken indefinitely small, the instantaneous axes of rotation along which sliding simultaneously takes place become indefinitely near each other; the motion is then a simple twist.

"Consider a pair of bodies having conical rolling, in which both cones have a motion of translation in space. The rotation then takes place through the conical rolling, and the sliding through the translation of the pair of bodies." Next "consider the consecutive positions of the axes as forming a pair of ruled surfaces, one for each body, so that the motion is reduced to the rolling of the two ruled surfaces upon each other with a simultaneous endlong sliding upon each other of the generators which are in contact. The surfaces of these solids being the loci of the axes, are called axoids. Thus all relative motions of two bodies may be considered as the twisting or rolling of ruled surfaces or axoids." The ruled surfaces roll on each other without sliding, when all the axes meet in a point as in a pair of cones or a cone and cylinder; also when the point of intersection is at any infinite distance, as in the case of two cylinders with parallel axes. These are, however, only particular and more obvious cases of the general condition of rolling without sliding, viz., that the two ruled surfaces are developable on each other.

\section{APPARATUS FOR REGISTERING ANIMAI MOVEMENTS 1}

THE registering apparatus which have enabled us to carzy so far the investigation of the functions in living animals are applicable to the analysis of movements of every kind in health and in disease. It is to this important application that $I$ desire to draw your attention at the present time.

Most of the movements whose various phases we have to estimate must be transmitted to a distance, preserving at the same time all their characteristics. It is by the medium of the air that this transmission is effected, and its principle is as follows :-

Upon the organ (muscle, artery, heart) whose movements are to be investigated an apparatus called the exploring drum is applied. It is a small metal basin closed by a caoutchouc membrane, and communicating by a longer or shorter tube with a similar drum, upon the membrane of which is supported a re cording lever. The pen with which the extremity of this lever is provided inscribes the curve of the movement impressed on the membrane of the first drum on a cylinder covered with smoked paper and turning on a horizontal axis.

I. Let us at once apply the process of analysis to the nuuscular movements of man. For this purpose we may either grasp the muscles of the ball of the thumb between the flattened jaws of the pincers which I show you, or apply to the fleshy substance of any muscle an exploring drum, the knob of which rests upon the muscle. When by means of electricity we cause a contraction or tetanus of the muscle to be studied, the curve of the contraction or that of the tetanus is recorded at a distance upon the revolving cylinder.

This apparatus shows the thickening which a muscle undergoes I Paper read in the Biological Section at the Loan Collection Conferences, 